\title{
Restauraciones protésicas sobre dientes con soporte periodontal reducido
}

\section{Prosthetic restoration on teeth with reduced periodontal tissue support}

\author{
Osorio Vélez LS*, Ardila Medina CM**
}

\section{RESUMEN}

Cuando se involucra a los pacientes con enfermedad periodontal en un plan de tratamiento integral, se pretende, en primera instancia, controlar la enfermedad. Sin embargo, superado este objetivo, se espera y se requiere el reemplazo protésico de los dientes perdidos así como la rehabilitación de los remanentes en la mayoría de los casos. Considerando que la elección de los dientes pilares para un restauración protésica depende de la capacidad biológica del periodonto sano reducido de soportar a largo plazo la restauración, numerosas investigaciones se han diseñado para establecer los criterios determinantes en tal selección.

Los resultados muestran que la principal limitación para el éxito de las restauraciones en pacientes con pocos pilares y una cantidad reducida de soporte periodontal, se relaciona con los factores biofísicos y técnicos y con el mantenimiento de la salud de los tejidos periodontales, más que con la cantidad de soporte periodontal.

Palabras clave: Restauraciones protésicas, periodonto reducido.

\section{SUMMARY}

When it is intended to provide an integral treatment plan for patients with periodontal disease; it is aimed, at first, to control the illness. Nevertheless, once this objective has been overcome, it is expected and required, the prosthetic replacement of lost teeth as well as rehabilitation for remaining ones, in most of cases. Considering that the selection of the abutments for a prosthetic restoration depends on the biological capacity of their healthy reduced periodontal tissues supporting the restoration in the long term; a lot of research has been carried to establish the determining criteria for such selection.

The results show that the limiting criteria for successful restorations in patients with few abutments and a reduced amount of periodontal support, is mainly related with biophysical and technical factors and with keeping healthy tissues, more than the periodontal support amount per se.

Key words: Prosthetic restoration, reduced periodontal tissue support.

Fecha de recepción: 11 de noviembre de 2008.

Aceptado para publicación: 25 de noviembre de 2008.

* Rehabilitadora Oral Universidad de Antioquía. Profesora Universidad Cooperativa de Colombia.

** Profesor Asistente Universidad de Antioquía. Presidente Sociedad Colombiana de Periodoncia-Regional Antioquía.

Osorio Vélez LS, Ardila Medina CM. Restauraciones protésicas sobre dientes con soporte periodontal reducido. Av. Odontoestomatol 2009; 25 (5): 287-293. 


\section{INTRODUCCIÓN}

Cuando se considera la historia natural de la enfermedad periodontal y su papel en la mortalidad dentaria, se pone de relieve dos situaciones altamente demandantes: la primera de ellas es que al final de la terapia periodontal activa, posiblemente solo algunos dientes podrán ser conservados, y la segunda, es que estos dientes remanentes, a menudo, presentan disminución del aparato de inserción y signos de movilidad. En la primera situación, se destaca la demanda de rehabilitación protésica en los dientes perdidos, pero la segunda situación, condiciona la decisión del clínico en la determinación de pilares protésicos adecuados para la rehabilitación.

La rehabilitación protésica es una parte importante del tratamiento integral de los pacientes con enfermedad periodontal avanzada. El objetivo de este artículo es establecer la capacidad biológica de un periodonto reducido saludable para soportar una restauración protésica a largo plazo, con base en la literatura científica disponible. Se presentarán además los desafíos protésicos periodontales y las implicaciones clínicas cuando se realizan restauraciones protésicas sobre pilares con periodonto disminuido.

\section{CAPACIDAD BIOLÓGICA DEL PERIODONTO REDUCIDO SALUDABLE PARA SOPORTAR UNA RESTAURACIÓN PROTÉSICA}

La investigación pionera de Nyman y Lindhe en 1979, en la que se evaluó el resultado del tratamiento periodontal y protésico en 299 pacientes con destrucción periodontal severa (pérdida igual o superior a $50 \%$ de soporte periodontal) y con restauraciones extensas, demostró que la salud periodontal puede mantenerse en pacientes comprometidos con un programa de higiene oral controlado, sin importar el diseño protésico efectuado en un paciente dado (1). Sin embargo, el uso de dientes con pequeñas cantidades de soporte periodontal como pilares para prótesis fija es controversial y contradice algunos parámetros extensamente acuñados en la literatura.

A partir del estudio de Jepsen (2), en el que especificó el área de superficie radicular promedio de los dientes humanos, y el de Ante (3) en el que se determinó que el área de superficie de los dientes pilares debe ser igual o superior a la de los dientes que reemplaza ("Ley de Ante") (4), se deduce que un diente perdido puede ser sustituido con éxito si los dientes pilares son periodontalmente saludables. $\mathrm{Si}$ se pierden dos dientes, una prótesis parcial fija probablemente puede reemplazarlos, pero si el área de superficie de los pónticos sobrepasa el de los pilares, la situación se consideraría inaceptable (5). Las anteriores presunciones, sugieren una limitación de la capacidad de soportar fuerzas masticatorias y de oclusión dada por el volumen del tejido periodontal medido en extensión de cubrimiento pericementario. Sin embargo, Laurell y colaboradores (6), investigaron el patrón de las fuerzas masticatorias y de oclusión dirigido axialmente en un grupo de pacientes tratados periodontalmente y restaurado con puentes con pilar terminal bilateral y en arco cruzado. En esta investigación, se instalaron transductores de deformación en los pónticos de manera que las fuerzas oclusales, actuando perpendicularmente al plano oclusal durante la función natural, podían ser simultáneamente estudiados en varias partes de la dentición. Los resultados demostraron que la capacidad del periodonto para soportar fuerzas transitorias solo se utilizó en forma parcial durante la masticación, con máxima resistencia en oclusión habitual ( $25 \%$ y $57 \%$ respectivamente) (6). Estos investigadores sugieren que el área de ligamento periodontal no limita la capacidad de soportar carga, sin embargo no descarta el que la module. De hecho, investigaciones posteriores, ha apuntado a que el ligamento periodontal proporciona una retroalimentación sensorial con respecto a la discriminación de fuerza de mordida (7).

Principalmente, en los casos de prótesis parcial fija (PPF), adherirse estrictamente a los conceptos de la denominada ley de Ante, limitaría el reemplazo protésico a condiciones de pilares ideales que no son la constante clínica, y mucho menos, en pacientes con compromiso periodontal. Es así, como algunas investigaciones proponen que dientes con periodonto reducido, pueden ser restaurados con éxito y considerados como pilares para reemplazo protésico, como lo ilustran Nyman y Ericsson (8). Estos autores concluyeron que en el $57 \%$ de las restauraciones, los dientes pilares tuvieron menos del 50\% del área 
de ligamento normal anticipado de los pónticos, y en solo el $8 \%$ de los casos, el área de ligamento de los pilares fue igual o excedió el de los pónticos, todas las prótesis habían funcionado apropiadamente durante 8-11 años. Además, los tejidos periodontales alrededor de los dientes pilares no había sufrido pérdida adicional de inserción durante el período de mantenimiento, independiente de que se aplicara un diseño de arco cruzado con pilares terminales bilaterales, unilaterales, cantilever bilateral o sin cruce de arco (8).

Freilich (9), evaluó el efecto de las PPF sobre dientes pilares móviles con soporte periodontal sustancialmente reducido, y los comparó con dientes control del mismo tipo y condición periodontal. No encontró diferencias entre ambos grupos, en un seguimiento realizado durante 24 meses (9).

Evaluaciones a más largo plazo como la de Yi y colaboradores (10), señalan un $86 \%$ de éxito en restauraciones en arco cruzado sobre dientes con destrucción periodontal avanzada, en donde los cambios longitudinales en la cantidad de soporte periodontal fueron mínimos, en un promedio de quince años de seguimiento, resultados que fueron independientes del diseño protésico. Estudios recientes y bien controlados, como el de Yi (11), corroboran los primeros hallazgos de Nyman, al evaluar 50 PPF cuyos pilares presentaban un promedio de solo $26 \%$ del tejido periodontal remanente y reemplazaban un $79 \%$ del área de ligamento total de los dientes. Los resultados de este estudio prospectivo a tres años, mostraron un cambio insignificante en el área del ligamento periodontal, aunque no se cumpliera la llamada Ley de Ante (11). De hecho, una investigación realizada en Arabia Saudita en 1997, concluyó que de las PPF construidas en el Collage of Dentistry en la Universidad de Arabia Saudita, 26.9\% no cumplían dicha ley y el 50\% de las realizadas en la práctica particular tampoco lo hacían. A pesar de ello, la evaluación radiográfica de los dientes pilares reveló que solo dos casos mostraron evidencia de sobrecarga de los pilares (12). No obstante, se tiene que reconocer que en todos los casos en los que se obtuvieron resultados satisfactorios en la rehabilitación protésica de pacientes con periodonto reducido, existió un programa estricto de mantenimiento y control. Es importante mencionar, que los estu- dios de Lövdal (13), Suomi (14), Ramflord (15, 16) Lyndhe-Nyman (17) y Rosling (18), han sustentado como la terapia mecánica, la eliminación de bolsas periodontales profundas y otros factores de retención de placa, así como la implementación de un programa adecuado de mantenimiento, eliminan los signos clínicos de la gingivitis y previenen un mayor deterioro de los tejidos de soporte.

Los elementos clave para un mantenimiento periodontal exitoso incluyen adecuada higiene oral personal y limpiezas profesionales efectuadas por personal calificado del área odontológica (19). Desafortunadamente, la mayoría de pacientes no eliminan efectivamente la placa bacteriana, por ello, el mantenimiento de una salud periodontal óptima requiere cuidado profesional (20), situación que es mas delicada si se tiene en cuenta que entre el $20 \%$ al $25 \%$ de los pacientes que cumplen con las recomendaciones de mantenimiento periodontal, puede ocurrir una pérdida significativa adicional a pesar del tratamiento (21). Por lo anterior, los programas de mantenimiento efectivo son esenciales para promover la continuidad de los objetivos logrados en el tratamiento, y permitirán evaluar además, los cambios de los perfiles clínicos y epidemiológicos de los pacientes (22).

\section{DESAFÍOS PROTÉSICOS}

Los pacientes periodontalmente comprometidos, forman parte de un grupo altamente demandante de soluciones protésicas que sean tanto estéticas como biomecánicamente compatibles con su soporte periodontal disminuido. En el caso de los pacientes parcialmente edéntulos, con soporte periodontal disminuido y que requieren un reemplazo protésico en su extensión distal, las opciones de tratamiento pueden incluir implantes, prótesis fija en cantilever y sobredentaduras (23).

Dentro de las indicaciones para la incorporación de prótesis parciales en cantilever, se encuentra la necesidad de incrementar la comodidad masticatoria, satisfacción de las demandas estéticas del paciente y dirigir apropiadamente las fuerzas en pacientes con soporte periodontal reducido en forma severa (24). Las investigaciones de Laurell y Lundgren (25-27), 
postulan que las fuerzas masticatorias recibidas por las restauraciones en cantilever son menores de los aproximadamente $700 \mathrm{~N}$ reportados en la literatura $(28,29)$, indicando también, que el lado restaurado con cantilever nunca constituye el lado preferente de masticación y que las fuerzas recibidas por tales restauraciones corresponden a solo la mitad o menos de lo que soportan con un pilar terminal distal. Este efecto se atribuye a la mayor flexibilidad y deflexión en la restauración con cantilever (26), lo cual sugiere un menor efecto de la medición del cubrimiento periodontal pericementario en la determinación de la capacidad de soporte de un diente en particular. Además, cuando se evalúa el efecto de tomar dientes pilares múltiples para restauración con cantilever en denticiones periodontalmente disminuidas, se observa que el efecto de distribución de fuerzas deja de ser significativo a partir del tercer pilar (30), descartando la necesidad de preparar protésicamente más dientes adicionales, hecho que solo contribuiría a disminuir la eficacia en la higiene oral, aumentando el costo biológico de la restauración.

En pacientes con periodonto reducido, la restauración fija se considera superior a la prótesis parcial removible, debido a que proporciona una distribución más favorable de las fuerzas de la masticación, al periodonto de los dientes pilares (1). Teniendo en cuenta que no se encuentran diferencias en los niveles de placa bacteriana y pérdida ósea adicional en aquellos dientes restaurados comparados con aquellos que no lo están (31), es posible considerar la PPF como un tratamiento adecuado en pacientes con periodonto reducido.

Aunque pueda lograrse eficiencia masticatoria y comodidad en los pacientes que usan restauraciones fijas o removibles, se encuentra que cuando se opta por la segunda opción se produce hasta seis veces más caries en los dientes pilares, en donde además, las condiciones funcionales y oclusales se deterioran en una mayor medida (32).

\section{DESAFÍOS PERIODONTALES}

Este tipo de retos incluye aspectos relacionados con la presencia de defectos óseos angulares y la realización de resecciones radiculares.
Con frecuencia, se presentan defectos óseos angulares profundos en dientes que se consideran pilares estratégicos para la restauración protésica. Las investigaciones $(30,33)$, demuestran que dichos defectos constituyen regiones localizadas de concentración preferencial de tensión y que entonces, las fuerzas oclusales, dependiendo de su magnitud y frecuencia, pueden exacerbar la situación en la región del defecto y posiblemente causar una pérdida ósea adicional. Los defectos óseos angulares han sido tratados con una variedad de acercamientos quirúrgicos diferentes que incluyen procedimientos resectivos y regenerativos $(34,35)$. Estudios a corto y largo plazo demuestran que la regeneración tisular guiada podría ser aplicada con éxito en el tratamiento de estos defectos alrededor de los pilares protésicos de manera que mejore el pronóstico de aquellos severamente comprometidos y puedan ser incluidos en las restauraciones definitivas $(36,37)$.

Las resecciones radiculares de dientes multirradiculares permite la preservación de dientes pilares de restauraciones fijas y removibles. Sin embargo, a pesar de que algunos autores han informado de buenos resultados $(38,39)$, cuando se evalúa el desempeño en el largo plazo se encuentran tasas de fracaso que van desde el 32\% (40) hasta el 38\% (41). A pesar de que la causa principal de fracaso fuese periodontal, es incierto si puede esperarse una alta incidencia de fracturas radiculares como lo sugieren algunos estudios $(41,42)$.

\section{IMPLICACIONES CLÍNICAS}

Se recomienda que los dientes pilares móviles, comprometidos periodontalmente, sean considerados para soportar PPF bajo las siguientes condiciones:

- Cuando se carece de otros pilares favorablemente distribuidos y con mayor soporte periodontal.

- Éxito comprobado de la terapia periodontal activa.

- Control de defectos óseos angulares.

- Reconsiderar dientes con procedimientos de resección radicular.

- Pacientes cuidadosamente seleccionados con respecto al cumplimiento del programa de mantenimiento. 
- Refinado esquema oclusal.

- Control o ausencia de parafunciones

Relacionado con este último aspecto, los estudios de Laurell y Lundgren (25-27), demuestran que el control de la oclusión en máxima resistencia depende del control muscular y no del periodonto, y que un periodonto reducido podría tener su soporte periodontal mecánicamente destruido si se somete a fuerzas intensas o indebidas como en el bruxismo y otras actividades parafuncionales.

\section{CONCLUSIONES}

1. Para cumplir el criterio de la ley de Ante, los odontólogos comúnmente aumentan el número de dientes pilares pero no hay fundamentación biológica y científica que soporte tal comportamiento clínico.

2. Este incremento en el número de retenedores puede, por el contrario, acelerar la falla de la restauración protésica al verse comprometido el espacio de los nichos y el acceso a la higiene oral.

3. A pesar de los resultados favorables en la mayoría de investigaciones sobre dientes con periodonto reducido, la elección de los dientes pilares sigue siendo una de los determinantes más críticos en el resultado de las restauraciones.

4. Ante la imposibilidad de establecer una regla matemática predecible (como posiblemente pretendía la ley de Ante) se hace más importante el criterio clínico y la selección cuidadosa del caso basada en el examen individual del paciente y la consideración de todas las particularidades del paciente.

5. En todo caso, la sola cuantificación del cubrimiento del ligamento periodontal no determina la conducta clínica y deben ser evaluados otros factores como esquema oclusal, características morfológicas del individuo que permiten predecir fuerza masticatoria, hábitos parafuncionales, historia de caries, compromiso y motivación del paciente, dentición antagonista, diagnóstico periodontal.

6. Puesto que la cantidad de fracasos de origen endodóntico es alta, puede ser muy importante hacer énfasis en este tipo de diagnóstico para los dientes pilares antes de comprometerlos en una rehabilitación protésica.

La prótesis periodontal ha resuelto muchos problemas para los pacientes con pérdida avanzada del periodonto y múltiple pérdida dentaria. Sin embargo, hay que reconocer que el éxito de los implantes oseointegrados ha tenido un efecto importante en el proceso de planeación del tratamiento periodontal $(43,44)$. En los casos en los que se considere tomar dientes en gran riesgo como pilares de prótesis, los implantes pueden constituir una alternativa apropiada para evitar actos "heroicos" de laboratorio dental y procedimientos clínicos arriesgados.

\section{BIBLIOGRAFÍA}

1. Nyman S, Lindhe J. A longitudinal study of combined periodontal and prosthetic treatment of patients with avanced periodontal disease. $\mathrm{J}$ Periodontol 1979;50:163-9.

2. Jepsen A. Root surface measurement and a method for $x$ - ray determination of root surface area. Acta odontol Scand 1963;21:35-46.

3. Ante IH. The fundamental principles of abutments. Mich State Dent Soc Bull 1926;8:14-23.

4. Johnston JF, Phillips RW, Dykema RW. Modern practice in crown and bridge prosthodontics, ed 3. Philadelphia, WB Saunders Co, 1971, p. 11.

5. Shillingburg, Fundamentos Esenciales de Prótesis Fija. 3 $3^{a}$ Ed. Quintessence, 2002.

6. Laurell L, Lundgren D. Periodontal ligament areas and occlusal forces in dentitions restored with cross-arch bilateral end abutment bridges. J Clin Periodontol 1985;12:850-60.

7. Williams WN, Low SB, Cooper WR, Cornell CE. The effect of periodontal bone loss on bite force discrimination. J Periodontol 1987;58:236-9.

8. Nyman S, Ericsson I. The capacity of reduced periodontal tissues to support fixed bridgework. J Clin Periodontol 1982;9:409-14. 
9. Freilich MA, Breeding LC, Keagle JG, Garnick JJ. Fixed partial dentures supported by periodontally compromised teeth. J Prosthet Dent 1991;65: 607-11.

10. Yi SW, Ericsson I, Carlsson GE, Wennström JL. Long-term follow-up of cross-arch fixed partial dentures in patients with advanced periodontal destruction. Evaluation of the supporting tissues. Acta Odontol Scand 1995;53:242-8.

11. Yi SW, Carlsson GE, Ericsson I. Prospective 3year study of cross-arch fixed partial dentures in patients with advanced periodontal disease. J Prosthet Dent 2001;86:489-94.

12. Fayyad MA, Al-Rafee MA. Failure of dental bridges. IV. Effect of supporting periodontal ligament. J Oral Rehabil 1997;24:401-3.

13. Lövdahl A, Arno A, Schei O, Waerhaug J. Combined effect of subgingival scaling and controlled oral hygiene on the incidence of gingivitis. Acta Odontol Scand 1961; 19:537-55.

14. Suomi JD, West JD, Chang JJ, McClendon BJ. The effect of controlled oral hygiene procedures on the progression of periodontal disease in adults: radiographic findings. J Periodontol 1971;42:562-4.

15. Ramfjord SP, Knowles JW, Nissle RR, Shick RA, Burgett FG.. Longitudinal study of periodontal therapy. J Periodontol 1973; 44:66-77.

16. Ramfjord SP, Knowles JW, Nissle RR, Burgett FG, Shick RA. Results following three modalities of periodontal therapy. J Periodontol 1975;46: 522-6.

17. Lyndhe J, Nyman S. The effect of plaque control and surgical pocket elimination on the establishment and maintenance of periodontal health $\mathrm{A}$ longitudinal study of periodontal therapy in cases of advanced disease. J Clin Periodont 1975;2: 67-79.

18. Rosling B, Nyman S, Lindhe J, Jern B. The healing potential of the periodontal tissues following different techniques of periodontal surgery in plaque-free dentitions. A 2-year clinical study. J Clin Periodontol 1976;3:233-50.

19. Soolari A. Compliance and its role in successful treatment o fan advanced periodontal case: Review of the literature and a case report. Quintessence Int 2002;33:389-96.

20. Wilson TG. Compliance. A review of the literature with possible applications to periodontics. J Periodontol 1987; 58:706-714.

21. Garrett S, Adams DF, Bogle G, Donly K, Drisko $\mathrm{CH}$, Hallmon WW et al. The effect of locally delivered controlled-released doxycycline or scaling and root planning on periodontal maintenance patients over 9 months. J Periodontol 2000;71:22-30.

22. Ardila CM. Fase de mantenimiento: Fundamentos y protocolo de mantenimiento. Rev Ustasalud Odont 2003;2:107-13.

23. Berg T. Maxillary distal-extension removible partial denture abutments with reduced periodontal support. J Prosthet Dent 1993;70:245-50.

24. Hämmerle C, Ungerer MC, Fantoni PC, Brägger U, Bürgin W, Lang NP. Long-term analysis of biologic and technical aspects of fixed partial dentares with cantilevers. Int J Prosthodont 2000; 13:409-15.

25. Laurell L, Lundgren D. Periodontal ligament areas and occlusal forces in dentitions restored with cross-arch unilateral posterior two-unit cantilever bridges. J Clin Periodontol 1986;13:33-8.

26. Laurell L, Lundgren D. Occlusal force during chewing and biting in dentitions restored with fixed bridges of cross-arch extension. II Unilateral posterior two-unit cantilevers. J Oral Rehabil 1986;13:191-203.

27. Laurell L, Lundgren D. Distribution of occlusal forces along unilateral posterior two-unit cantilever segments in cross-arch fixed partial dentures. J Prosthet Dent 1988;60:106-112 
28. Erhardsson S. Fracture mechanics design of dental gold soldered joints (thesis). Swed Dent J 1980;suppl 5:16-8.

29. Glantz PO, Nyman S, Strandman E, Randow K. On functional strain in fixed mandibular reconstructions. II. An in vivo study. Acta Odontol Sand 1984;42:269-76.

30. Wylie R.S. Fixed cantilever splints on teeth with normal and reduced periodontal support. J Prosthet Dent 1991;66:737-42.

31. Valderhaug J. Oral higiene, periodontal conditions and carious lesions in patients treated with dental bridges. A 15-year clinical and radiographic follow-up study. J Clin periodontol 1993;20: 482-9.

32. Budtz-Jorgensen, Isidor F. A 5-year longitudinal study of cantilevered fixed partial dentures compared with removable partial dentures in a geriatric population. J Prosthet Dent 1990;64: 42-7

33. Fujita T. Photoelastic stress análisis of occlusal force distribution with periodontally envolved teeth. J Dent Res 1980;59.

34. Palkanis K. Surgical pocket therapy. In Genco R, editor. World workshop in periodontics. Lansdowne: American Academy of Periodontology1996:589617.

35. Garret S. Peridontal regeneration around natural teeth. In Genco R, editor. World workshop in periodontics. Lansdowne: American Academy of Periodontology; 1996:621-66.

36. Tonetti MS, Pini Prato G, Stalpers G, Cortellini P. Guided tissue regeneration of deep intrabony defects in strategically important prosthetic abutments. Int J Periodont Rest Dent 1996;16: 378-87.
37. Cortellini P, Stalpers G, Pini Prato G, Tonetti MS. Long-term clinical outcomes of abutments treated with guided tissue regeneration. J Prosthet Dent 1999;81:305-11.

38. Bergenholz A. Radectomy of multirooted teeth. J Am Dent Assoc 1972;85:870-5.

39. Hamp SE et al. Periodontal treatment of mulrirooted teeth. Results after 5-years. J Clin Periodontol 1975; 2:126-35.

40. Bühler H. Evaluation of root-resected teeth. Results after 10 years. J Periodontol 1988; 59(12): 805-10.

41. Langer B, Stein SD, Wagenberg B. An evaluation of root resections. A ten-year study. J Periodontol 1981;52:719-22.

42. De Backer H, Van Maele G, De Moor N, Van den Berghe L, De Boever J. A 20-year retrospective survival study of fixed partial dentures. Int $\mathrm{J}$ Prosthodont 2006; 19:143-53

43. Nevins Myron. Periodontal prosthesis reconsidered. Int J Prosthodont 1993;6:209-17.

44. Cordaro L, Ercoli C, Rossini C, Torsello F, Feng C. Retrospective evaluation of complete-arch fixed partial dentures connecting teeth and implant abutments in patients with normal and reduced periodontal support. J Prosthet Dent 2005;94:313-20.

\section{CORRESPONDENCIA}

Carlos Martín Ardila Medina

Universidad de Antioquía.

Carrera 47 No. 20 sur 46 Envigado Antioquía 57(4) 3348122

cmartin@odontologia.udea.edu.co 